\title{
Investigating flow in MHD micropumps
}

\author{
Saied Moghaddam ${ }^{1}$ D
}

Received: 26 August 2019 / Accepted: 6 November 2019 / Published online: 13 November 2019

(c) Springer Nature Switzerland AG 2019

\begin{abstract}
An analytical solution is found for assessing the performance of a DC- and AC-operated MHD micropump. The test fluid is assumed to be incompressible and Newtonian. The flow of MHD micropump is generated by the Lorentz force and is considered to be unidirectional, laminar and two-dimensional. The effect of Hartman number and channel's width is studied on the volumetric flow rate. It is concluded that there is an optimum Hartmann number and optimum aspect ratio for maximizing volumetric flow rate. A simple relationship is obtained for the optimum Hartman number which maximizes the flow rate. A comparison with known numerical solution and also available experimental data supports the validity of the analytical solution found in this work. Also the maximum volumetric flow rate, optimum Hartman number and optimum channel's width of DC-operated and AC-operated MHD micropump are compared together.
\end{abstract}

Keywords MHD micropump $\cdot$ Rectangular channel $\cdot$ Hartmann number $\cdot$ Lorentz force $\cdot$ Analytical solution

\section{Introduction}

An extraordinary assortment of techniques have been created over the years to pump liquids in microsystems [1]. Among them, dynamic micropumps, which apply powers straightforwardly on the fluid without having any moving part, appear to have a better future. A promising type of micropump which falls into this category is the magnetohydrodynamic (MHD) one [2]. These pumps rely on the interaction between a transverse magnetic field and an externally-imposed electrical current for moving an electrically-conducting liquid forward by the rise of the perpendicular force to both the electric and the magnetic fields. This force is called Lorentz force. Possible applications for such micropumps are spreading drug into the body, heat transfer in microelectronic equipment, analyzing of biological and chemical parameters by distributing liquids in microsystems, and more. In recent years they are increasingly being used in microfluidic and micro-electromechanical systems (MEMS) where space limitation and size are major concerns [2].
There are as of now two unique versions of MHD micropumps in current use: DC-operated, and AC-operated. The previous depends on a permanent magnet to produce the Lorentz force [3] while in the last an electromagnet is used [4]. The DC version suffers from the problem that by operating DC voltage in aqueous solutions, water is electrolyzed and therefore bubbles are formed. Because in the majority of MHD micropumps the electrodes are directly connected to the pumping channel, the bubbles generation can indeed be very problematic. Another problem of DC-operated MHD micropumps is Faradaic reactions which cause electrodes to degrade $[5,6]$. An AC-operated MHD micropump eliminates these two problems. For this reason, it appears to have a brighter future even though it has a lower efficiency (because notwithstanding the consumption of power by the electric current in the channel, the power expected to make the electromagnet should also be taken into account). The AC-operated MHD micropump relies on a perpendicular current and magnetic fields both oscillating at the same frequency in order to generate a rather pulsating flow. The utilization of $\mathrm{AC}$ voltage diminishes the electrolytic reactions

Saied Moghaddam, saiedmoghaddam@ut.ac.ir | ${ }^{1}$ Department of Food Technology, College of Aburaihan, University of Tehran, Tehran, Iran. 
which is necessary to retain the electric current. As a result, degradation of electrode and gas bubbles generation are eliminated. In contrast, the oscillating magnetic field generates eddy current through Faraday's law which can be troublesome when pumping liquid metals [7-9]. In the DCoperated MHD micropump, the electrolytic reaction which is responsible for current passing likewise generates gas bubbles which obstruct liquid stream and cause electrodes dissolution. This confines the functional use of DC-operated MHD micropump to non-electrolytic conducting liquids, like liquid metals. At the point by passing of an AC current in the electrolytic solution, with adequately high frequency, causes the chemical reactions to reverse with a rapid rate and bubbles never get an opportunity to produce and no degradation of electrode can happen.

The DC- and AC-operated MHD micropumps have both been the subject of much study in the last decade [10]. In addition to the experimental investigations, there have been extensive studies in the theoretical and/or numerical domains to address the parameters affecting their performance [11-25]. The main objectives of these studies have been to estimate the maximum flow rate and pressure of the micropump, the power needed and energy efficiency, and also the range of conductivity that the micropump can actuate. Issues influencing the most common features of micropumps like generation of heat, variation of liquid's physical properties, and bubble production by electrochemical reactions have also been explored in prior studies [11-25]. Due to the complexity of the governing equations, analytical solutions cannot, generally-speaking, be obtained for MHD micropumps. This is perhaps why most studies in this area are devoted to numerical methods. One can mention, for example, the numerical work by Wang et al. [26] in which the effects of channel's geometry has been investigated numerically in AC-operated MHD micropums. Assuming the flow to be steady, laminar, unidirectional, and two-dimensional, they showed that the channel dimensions have a strong influence on the channel's flow rate.

In the present work we will try to find an analytical solution for the AC- and DC-operated MHD micropump and compare these two kinds of micropumps. The effects of Hartman number and channel's width on the pump's performance is studied on the volumetric flow rate. Finally, a relation, each, has been found for optimum Hartmann number and optimum aspect ratio which maximize volumetric flow rate that had not been addressed before.

\section{Configurations and problem formulation}

Consider an electrically conducting incompressible Newtonian fluid which flows in an MHD micropump at fullydeveloped laminar condition. Figure 1 shows the problem's geometry. An external magnetic and electric fields are forced to a rectangular duct having a dimensions of $w$ (width), $h$ (height), and $L$ (total length). A voltage difference of magnitude $2 \mathrm{~V}$ forced across two opposing electrodes of channel (having a length of $L_{p}$ ) which generates an electric current density (shown by $\mathrm{J}$ ) in the fluid. A uniform and constant magnetic flux density $B$ is placed in the direction of $y$ axis (see Fig. 1).

The interaction between the magnetic field and the electric field imposes a force (perpendicular to both fields) which named as Lorentz force. This force causes the liquid move ahead in the direction of $x$. The governing equations of the fluid flow in a duct are completely complicated in its most general form. For simplicity, like Wang et al. [26], it is assumed that the components of velocity in the directions of $y$ and $z$ axis are small in comparison with the velocity along the $x$ direction, i.e., in the axis of conduit. The equation of continuity then reduces to $\partial u / \partial x=0$. The equation of $x$-momentum in Navier-Stokes equations also simplifies to:

$\rho \frac{\partial u}{\partial t}=-\frac{\partial p}{\partial x}+\mu \frac{\partial^{2} u}{\partial y^{2}}+\mu \frac{\partial^{2} u}{\partial z^{2}}$

where $\rho$ is the density and $\mu$ defines the viscosity of the conducting fluid. The equations of $y$ - and $z$-momentum in Navier-Stokes equations simplifies to $\frac{\partial p}{\partial y} \approx 0$ and $\frac{\partial p}{\partial z} \approx 0$. The cross product for the vectors of the electric current density and the density of the magnetic field results in a pressure gradient leading to the Lorentz force.

\subsection{Dc-operated mhd micropump}

For DC-operated, the pressure gradient so produced can be composed as [26]:

$\Delta p=(\vec{J} \times \vec{B}) L_{p}$

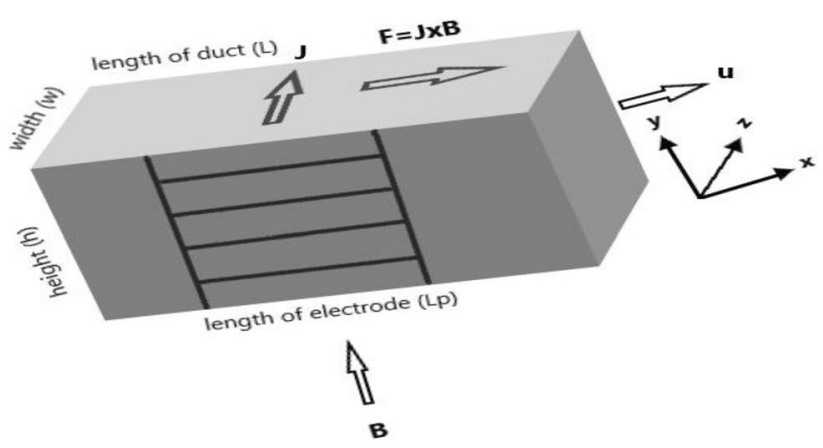

Fig. 1 Schematic view of the MHD micropump geometry and coordinate system 
where relations of $\vec{B}$ and $\vec{J}$ are coupled together by the Ohm's law [26]:

$\vec{J}=\sigma(\vec{E}+\vec{u} \times \vec{B})$

In the above equation, $\sigma$ and $\vec{E}$ are the electrical conductivity and the electric field intensity. The term $\vec{J} \times \vec{B}$ in Eq. 2 is named as Lorentz force. We also have:

$\vec{E}=\frac{2 V}{w}$

Assuming that the distribution of pressure difference is uniform over the whole channel length, the pressure gradient in Eq. 1 is replaced by:

$-\frac{\partial p}{\partial x}=\frac{\Delta p}{L}$

With this little maneuvering, Eq. 1 becomes:

$\rho \frac{\partial u}{\partial t}=\mu \frac{\partial^{2} u}{\partial y^{2}}+\mu \frac{\partial^{2} u}{\partial z^{2}}-\frac{L_{p} \sigma B^{2}}{L} u+\frac{2 L_{p} \sigma B V}{L w}$

Solving this equation need the boundary conditions. They are as below:

$u(0, z, t)=u(h, z, t)=0$

$u(y, 0, t)=u(y, w, t)=0$

And the initial condition is given by:

$u(y, z, 0)=0$

Having determined the velocity profile at any points of $y$ - and $z$-location, the volumetric flow rate is obtained as:

$Q=\int_{0}^{w} \int_{0}^{h} u(y, z, t) d y d z$

Prior to attempting to solve the governing equation, Eq. 6 , it is helpful to obtain the above mentioned equations in dimensionless form. So the following dimensionless parameters and variables are defined:

$$
\begin{aligned}
\tau & =\frac{u_{0} t}{L}, y^{*}=\frac{y}{L^{\prime}} z^{*}=\frac{z}{L^{\prime}}, u^{*}=\frac{u}{u_{0}}, \alpha=\frac{h}{L}, \beta=\frac{w}{L}, L_{p}^{*}=\frac{L_{p}}{L} \\
H a & =L B \sqrt{\frac{\sigma}{\mu}}, V^{*}=\frac{V}{u_{0}} \sqrt{\frac{\sigma}{\mu}}, \operatorname{Re}=\frac{\rho u_{0} L}{\mu}, Q^{*}=\frac{Q}{u_{0} L^{2}}
\end{aligned}
$$

where $u_{0}$ is a characteristic velocity (it can be the average velocity). In dimensionless form, Eq. 6 becomes:

$\operatorname{Re} \frac{\partial u^{*}}{\partial \tau}=\frac{\partial^{2} u^{*}}{\partial y^{*^{2}}}+\frac{\partial^{2} u^{*}}{\partial z^{*^{2}}}-L_{p}^{*} H a^{2} u^{*}+\frac{2 L_{p}^{*} H^{*} V^{*}}{\beta}$
The dimensionless boundary conditions become:

$u^{*}\left(0, z^{*}, \tau\right)=u^{*}\left(\alpha, z^{*}, \tau\right)=0$

$u^{*}\left(y^{*}, 0, \tau\right)=u^{*}\left(y^{*}, \beta, \tau\right)=0$

The dimensionless initial condition becomes:

$u^{*}\left(y^{*}, z^{*}, 0\right)=0$

As to the non-dimensional volumetric flow rate we have:

$Q^{*}=\int_{0}^{\beta} \int_{0}^{\alpha} u^{*}\left(y^{*}, z^{*}, \tau\right) d y^{*} d z^{*}$

\subsection{Ac-operated mhd micropump}

The similar methodology as done DC-operated MHD micropump has also been utilized to obtain equation of the AC-operated MHD micropump. In this condition, the equations of pressure gradient, current density and electric field intensity are: $\Delta p=[\vec{J} \times \vec{B} \sin (\omega t)] L_{p}$ ,$\vec{J}=\sigma[\vec{E}+\vec{u} \times \vec{B} \sin (\omega t)]$, and $\vec{E}=2 V \sin (\omega t+\phi) / w$, where $\omega$ and $\phi$ are the frequency and the phase angle between the magnetic and electric fields. The equation of momentum in fully developed flow of AC-operated MHD micropump is then:

$$
\begin{aligned}
\operatorname{Re} \frac{\partial u^{*}}{\partial \tau}= & \frac{\partial^{2} u^{*}}{\partial y^{*^{2}}}+\frac{\partial^{2} u^{*}}{\partial z^{*^{2}}}-L_{p}^{*} H a^{2} \sin ^{2}\left(\omega^{*} \tau\right) u^{*} \\
& +\frac{2 L_{p}^{*} H a V^{*}}{\beta} \sin \left(\omega^{*} \tau\right) \sin \left(\omega^{*} \tau+\phi\right)
\end{aligned}
$$

where $\omega^{*}=\frac{L \omega}{u_{0}}$.

Details of analytical solution applied for MHD micropump will be produced in the next section.

\section{Analytical solution}

\subsection{Dc-operated mhd micropump}

The eigenfunction expansion method can be used to solve the governing equation, Eq. 11. As an initial step, consider first the eigenfunctions of the homogeneous problem of Eq. 11:

$\operatorname{Re} \frac{\partial u^{*}}{\partial \tau}=\frac{\partial^{2} u^{*}}{\partial y^{*^{2}}}+\frac{\partial^{2} u^{*}}{\partial z^{*^{2}}}-L_{p}^{*} H a^{2} u^{*}$

$u^{*}\left(0, z^{*}, \tau\right)=u^{*}\left(\alpha, z^{*}, \tau\right)=0$

$u^{*}\left(y^{*}, 0, \tau\right)=u^{*}\left(y^{*}, \beta, \tau\right)=0$ 
Since Eq. 16 and its boundary conditions are homogeneous and linear, we resort to the method of separationof-variables for finding a solution:

$u^{*}\left(y^{*}, z^{*}, \tau\right)=\delta(\tau) \psi\left(y^{*}, z^{*}\right)$

After deriving the derivatives of Eq. 18 and putting them into Eq. 16, below equation obtains:

$\frac{\operatorname{Re} \frac{\partial \delta}{\partial \tau}+L_{p}^{*} H a^{2} \delta}{\delta}=\frac{\frac{\partial^{2} \psi}{\partial y^{* 2}}+\frac{\partial^{2} \psi}{\partial z^{*^{2}}}}{\psi}=-\lambda^{2}$

where $\lambda^{2}$ is a constant. From this equation, the two-dimensional eigenvalue problem becomes:

$\left(\frac{\partial^{2} \psi}{\partial y^{*^{2}}}+\frac{\partial^{2} \psi}{\partial z^{*^{2}}}\right)+\lambda^{2} \psi=0$

$\psi\left(0, z^{*}\right)=\psi\left(\alpha, z^{*}\right)=0$

$\psi\left(y^{*}, 0\right)=\psi\left(y^{*}, \beta\right)=0$

Equation 20 with its boundary conditions, Eq. 21, can be solved by the "separation-of-variables" method to yield the eigenfunctions as:

$\psi_{m n}\left(y^{*}, z^{*}\right)=\sin \left(\frac{n \pi}{\alpha} y^{*}\right) \sin \left(\frac{m \pi}{\beta} z^{*}\right)$

And the eigenvalues are:

$\lambda_{m n}^{2}=\left(\frac{n \pi}{\alpha}\right)^{2}+\left(\frac{m \pi}{\beta}\right)^{2}$

where $m=1,2, \ldots, \infty$ and $n=1,2, \ldots, \infty$ Now consider that non-homogeneous Eq. 11 has a series-solution based on the Eq. 24:

$u^{*}\left(y^{*}, z^{*}, \tau\right)=\sum_{m=1}^{\infty} \sum_{n=1}^{\infty} c_{m n}(\tau) \psi_{m n}\left(y^{*}, z^{*}\right)$

Substituting $u^{*}\left(y^{*}, z^{*}, \tau\right)$ and its derivatives from Eq. 24 into Eq. 11 obtains:

$\sum_{m=1}^{\infty} \sum_{n=1}^{\infty}\left(\operatorname{Re} \frac{d c_{m n}}{d \tau}+\left(L_{p}^{*} H a^{2}+\lambda_{m n}^{2}\right) c_{m n}(\tau)\right) \psi_{m n}\left(y^{*}, z^{*}\right)=\frac{2 L_{p}^{*} H a V^{*}}{\beta}$

where from initial conditions, we have: $c_{m n}(0)=0$. Equation 25 is a double Fourier series. For solving $c_{m n}(\tau)$, let

$\Lambda_{m n}=\operatorname{Re} \frac{d c_{m n}}{d \tau}+\left(L_{p}^{*} \mathrm{Ha}^{2}+\lambda_{m n}^{2}\right) c_{m n}(\tau)$
This yields:

$\sum_{m=1}^{\infty} \sum_{n=1}^{\infty} \Lambda_{m n} \psi_{m n}\left(y^{*}, z^{*}\right)=\frac{2 L_{p}^{*} \mathrm{HaV}^{*}}{\beta}$

Then $\Lambda_{m n}$ obtains as:

$\Lambda_{m n}=\left\{\begin{array}{cc}\frac{32 L_{p}^{*} H a V^{*}}{\beta m n \pi^{2}}, & \mathrm{~m} \text { and } \mathrm{n} \text { odd } \\ 0, & \mathrm{~m} \text { or } \mathrm{n} \text { even }\end{array}\right.$

Equation 26 is an ODE (ordinary differential equation) of first-order, and can be solved to give:

$c_{m n}(\tau)=\frac{32 L_{p}^{*} H a V^{*} \cos \phi}{\beta m n \pi^{2}\left(\lambda_{m n}^{2}+L_{p}^{*} H a^{2}\right)}\left(1-e^{\left(-\frac{\left(\lambda_{m n}^{2}+L_{p}^{*} H a^{2}\right)}{R e} \tau\right)}\right)$

From Eq. 14, volumetric flow rate can be written as:

$Q^{*}(\tau)=\sum_{m=1}^{\infty} \sum_{n=1}^{\infty} c_{m n}(\tau) K_{m n}$

where $K_{m n}$ is:

$K_{m n}=\left\{\begin{array}{cc}\frac{4 \alpha \beta}{m n \pi^{2}}, & m \text { and } \mathrm{n} \text { odd } \\ 0, & \mathrm{~m} \text { or } \mathrm{n} \text { even }\end{array}\right.$

\subsection{Ac-operated mhd micropump}

The identical method as made above has also been successfully drawn to obtain an analytical solution for the AC-operated version of MHD micropump. After using the method of separation-of-variables the below equation is found for the velocity of AC MHD micropump:

$u^{*}\left(y^{*}, z^{*}, \tau\right)=\sum_{m=1}^{\infty} \sum_{n=1}^{\infty} c_{m n}^{\prime}(\tau) \psi_{m n}\left(y^{*}, z^{*}\right)$

where $\psi_{m n}\left(y^{*}, z^{*}\right)$ is defined in Eq. 22 and $c_{m n}^{\prime}(\tau)$ should be found from Eq. 33 .

$\Lambda_{m n}^{\prime}=\operatorname{Re} \frac{d c_{m n}}{d \tau}+\left(L_{p}^{*} H a^{2} \sin ^{2}\left(\omega^{*} \tau\right)+\lambda_{m n}^{2}\right) c_{m n}(\tau)$

where $\Lambda_{m n}^{\prime}$ is defined as:

$\Lambda_{m n}^{\prime}=\left\{\begin{array}{cc}\frac{32 L_{p}^{*} H a V^{*}}{\beta m n \pi^{2}} \sin \left(\omega^{*} \tau\right) \sin \left(\omega^{*} \tau+\phi\right), & \mathrm{m} \text { and } \mathrm{n} \text { odd } \\ 0, & \mathrm{~m} \text { or } \mathrm{n} \text { even }\end{array}\right.$

Equation 33 is an ODE (ordinary differential equation) of first order, and can be solved to give: 
$C_{m n}^{\prime}(\tau)=\frac{32 L_{p}^{*} H a V^{*}}{\operatorname{Re} \beta m n \pi^{2}} \times \frac{\left.\int_{0}^{\tau} e^{\left(\frac{\left(\lambda_{m n}^{2}+\frac{L_{p}^{*}+a^{2}}{2}\right)}{\operatorname{Re}} \tau-\frac{L_{p}^{*} H A^{2}}{4 \omega^{*} \mathrm{Re}} \sin \left(2 \omega^{*} \tau\right)\right.}\right) \sin \left(\omega^{*} \tau\right) \sin \left(\omega^{*} \tau+\phi\right) d \tau}{\left.e^{\left(\frac{\left(\lambda_{m n}^{2}+\frac{L_{p}^{*} H a^{2}}{2}\right)}{\mathrm{Re}} \tau-\frac{L_{p}^{*} H \alpha^{2}}{4 \omega^{*} \mathrm{Re}} \sin \left(2 \omega^{*} \tau\right)\right.}\right)}$

Values of $\omega^{*}$ in more real MHD micropump systems are large [4], so in this case because of $\frac{\left(\lambda_{m n}^{2}+\frac{L_{p}^{*} H a^{2}}{2}\right)}{\operatorname{Re}} \tau>>\frac{L_{p}^{*} H a^{2}}{4 \omega^{*} \operatorname{Re}} \sin \left(2 \omega^{*} \tau\right)$, the term of $\frac{L_{p}^{*} H a^{2}}{4 \omega^{*} \mathrm{Re}} \sin \left(2 \omega^{*} \tau\right)$ can be neglected in the above equation. Finally the following relationship for $c_{m n}^{\prime}(\tau)$ can be concluded:

$$
\begin{aligned}
& c_{m n}^{\prime}(\tau) \cong \frac{16 L_{p}^{*} H a V^{*}}{\operatorname{Re} \beta m n \pi^{2}} \times\left[\frac{\operatorname{Re} \cos \phi}{\lambda_{m n}^{2}+\frac{L_{p}^{*} H a^{2}}{2}}\left(1-e^{\left(-\frac{\left(\lambda_{m n}^{2}+\frac{L_{p}^{*} H a^{2}}{2}\right)}{\operatorname{Re}} \tau\right)}\right)\right.
\end{aligned}
$$

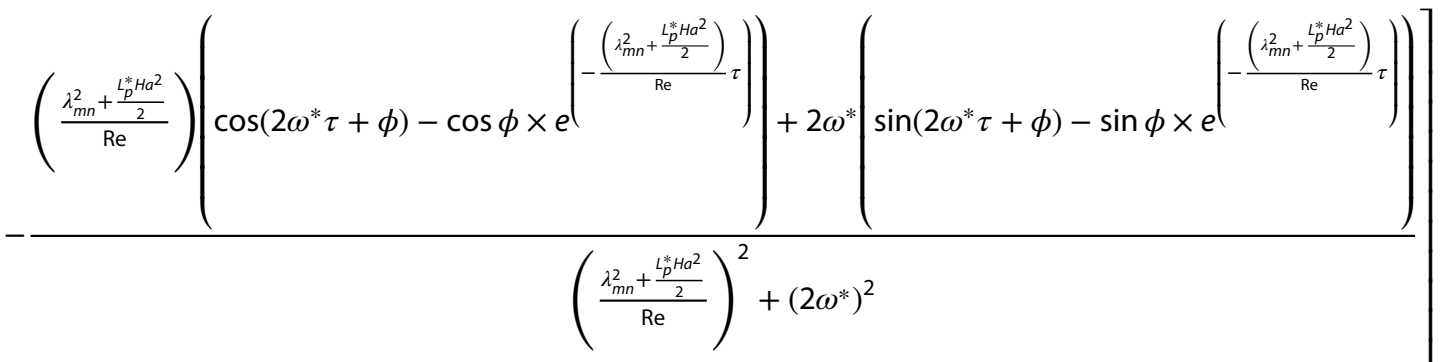

\section{Main results}

\subsection{Validation}

In this section we will check the performance of our analytical solution of DC-operated MHD micropump using the experimental results reported in Ref. 11 and the solution of AC-operated MHD micropump by the numerical where the second term of the outermost bracket in Eq. 36 goes to zero for large values of $\omega^{*}$. So $c_{m n}^{\prime}(\tau)$ can be written as below:

$c_{m n}^{\prime}(\tau)=\frac{16 L_{p}^{*} H a V^{*} \cos \phi}{\beta m n \pi^{2}\left(\lambda_{m n}^{2}+\frac{L_{p}^{*} H a^{2}}{2}\right)}\left(1-e^{\left(-\frac{\left(\lambda_{m n}^{2}+\frac{L_{p}^{*} H a^{2}}{2}\right)}{R e} \tau\right)}\right)$

Also volumetric flow rate is:

$Q^{*}(\tau)=\sum_{m=1}^{\infty} \sum_{n=1}^{\infty} c_{m n}^{\prime}(\tau) K_{m n}$

where $K_{m n}$ is defined in Eq. 31. results reported in Ref. 26 and the experimental results reported in Ref. 4.

\subsubsection{Dc-operated mhd micropump}

We set $\mathrm{w}=3 \mathrm{~mm}$ (width of channel), $\mathrm{h}=7 \mathrm{~mm}$ (height of channel), $L=80 \mathrm{~mm}$ (length of channel), and the $L_{p}=35 \mathrm{~mm}$ (length of the electrodes). The viscosity of working fluid is 0.0006 Pa.s, and its electrical conductivity is equal to $1.5 \mathrm{~s} / \mathrm{m}$. Also, the density of magnetic flux is fixed at $0.02 \mathrm{~T}$. The flow rate of the present analytical results with the experimental results reported in Ref. 11 are compared in Fig. 2. Results show a good agreement between analytical and experimental results and their maximum relative error is not more than $12 \%$. 


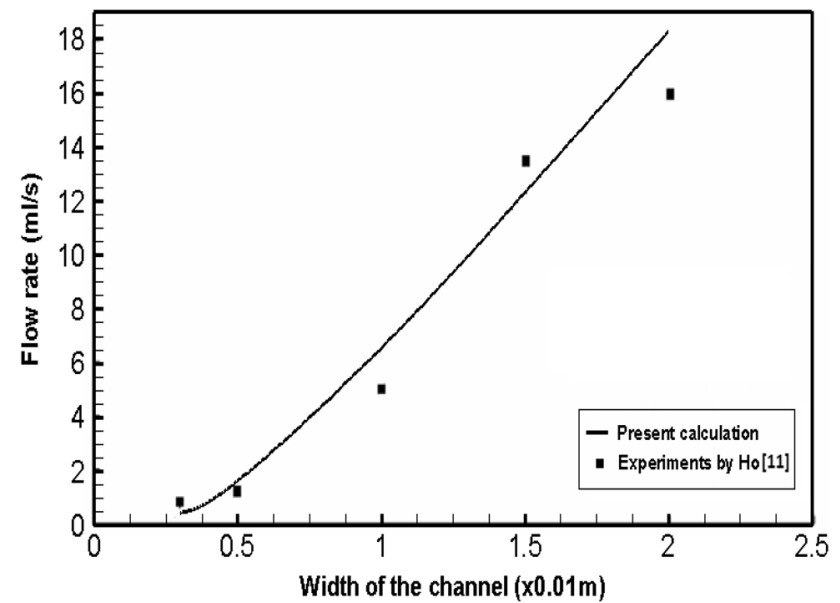

Fig. 2 Comparison the flow rate of present solution and experimental data for DC-operated MHD micropump

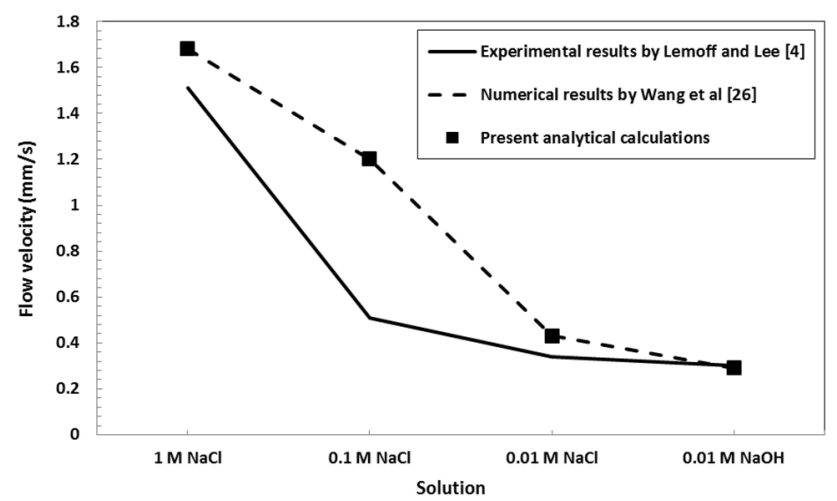

Fig. 3 Comparison the flow velocity of present analytical solution with the experimental and numerical results for AC-operated MHD micropump

\subsubsection{Ac-operated mhd micropump}

We set $\mathrm{w}=800 \mu \mathrm{m}$ (width of channel), $\mathrm{h}=380 \mu \mathrm{m}$ (height of channel), $L=20 \mathrm{~mm}$ (length of channel), $\mathrm{L}_{\mathrm{p}}=4 \mathrm{~mm}$ (length of the electrodes) and $\omega=1 \mathrm{kHz}$ (frequency). Saline solutions have been utilized as the test fluid. The viscosity of $\mathrm{NaCl}$ solutions is 0.0006 Pa.s, and its electrical conductivity is $1.5 \mathrm{~s} / \mathrm{m}$. The average density of $A C$ magnetic flux is fixed at $13 \mathrm{mT}$, and different electric currents with values of $24,36,100$, and $140 \mathrm{~mA}$ are applied. Figure 3 shows a comparison between the present analytical results with the numerical results [26] and experimental results [4] under steady conditions. A satisfactory coincidence is seen to exist between experimental and analytical results except in case of $0.1 \mathrm{M} \mathrm{NaCl}$. As mentioned in Ref. 4, there are experimental errors for case of $0.1 \mathrm{M} \mathrm{NaCl}$, therefore present calculations and experimental data are very different with together. Also results of present

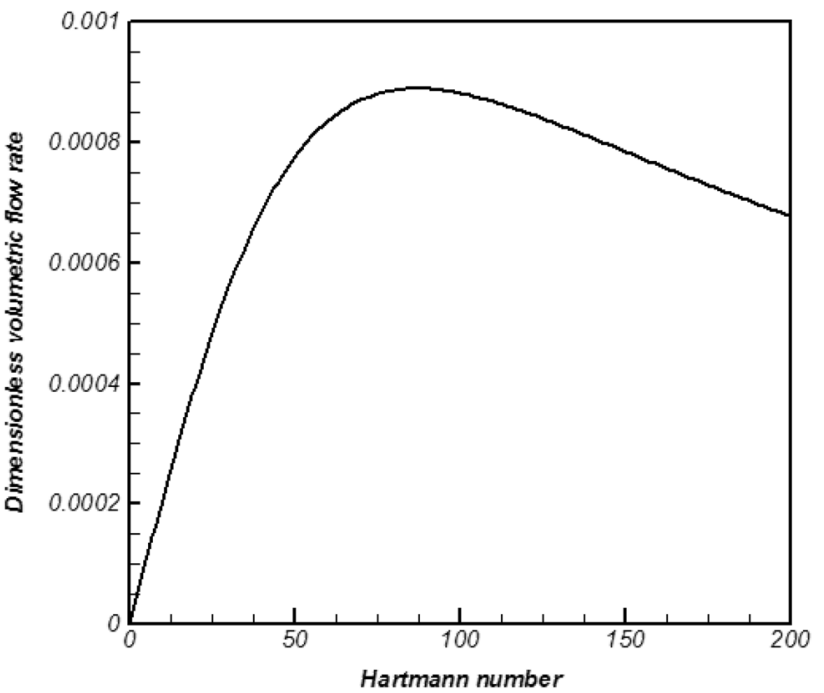

Fig. 4 Effect of the Hartmann number on the volumetric flow rate of DC-operated MHD micropump $\left(\alpha=0.1, \beta=0.1, L_{p}^{*}=0.3, V^{*}=1\right)$

analytical solution and numerical solution [26] are exactly same together.

Having verified the analytical solution, it will be used to investigate the effect of Hartmann number and channel's width on the volumetric flow rate.

\subsection{Hartmann number effect}

\subsubsection{Dc-operated mhd micropump}

Figure 4 displays the impact of the Hartmann number on the volumetric flow rate profile. Based on this figure, volumetric flow rate becomes maximum for an optimum Hartmann number.

As a matter of fact, our analytical solution should enable one to find a closed-form solution for the optimum Hartmann number. To that end, we take the derivative of Eq. 30 as

$\frac{d Q_{\text {steady }}^{*}}{d \mathrm{Ha}}=0$

and obtain,

$\sum_{m=1}^{\infty} \sum_{n=1}^{\infty} \frac{\lambda_{m n}^{2}-L_{p}^{*} H a^{2}}{(m n)^{2}\left(\lambda_{m n}^{2}+L_{p}^{*} H a^{2}\right)^{2}}=0, \mathrm{~m}$ and $\mathrm{n}$ odd

After mathematical calculation we can derive the below equation: 
$\sum_{m=1}^{\infty} \sum_{n=1}^{\infty} \frac{\left(\frac{n \pi \beta}{\alpha}\right)^{2}+(m \pi)^{2}-\left(\beta \sqrt{L_{p}^{*} H a}\right)^{2}}{(m n)^{2}\left(\left(\frac{n \pi \beta}{\alpha}\right)^{2}+(m \pi)^{2}+\left(\beta \sqrt{L_{p}^{*} H a}\right)^{2}\right)^{2}}=0, \quad m$ and $\mathrm{n}$ odd

Then we can find the relation of optimum Hartmann number as below:

$\left(H a_{o p t}\right)_{D C}=\frac{1}{\beta \sqrt{L_{p}^{*}}} g\left(\frac{\alpha}{\beta}\right)$

Using numerical solution we can find $g\left(\frac{\alpha}{\beta}\right)$ which is plotted in Fig. 5a. Substituting Eq. 42 into Eq. 30 yields the maximum flow rate as:

$Q_{\max }^{*}=V^{*} \sqrt{L_{p}^{*}} \alpha \beta f\left(\frac{\alpha}{\beta}\right)$

where $f\left(\frac{\alpha}{\beta}\right)$ is plotted in Fig. $5 b$.

We can also define the efficiency of DC-operated MHD micropumps by:

$\eta\left(\frac{H a}{\left(H a_{o p t}\right)_{D C}}\right)=\frac{Q^{*}}{Q_{\max }^{*}}$

where $\eta\left(\frac{H a}{\left(H a_{o p t}\right)_{D C}}\right)$ is plotted in Fig. 6.

Finally, the relation of steady volumetric flow rate is obtained as:

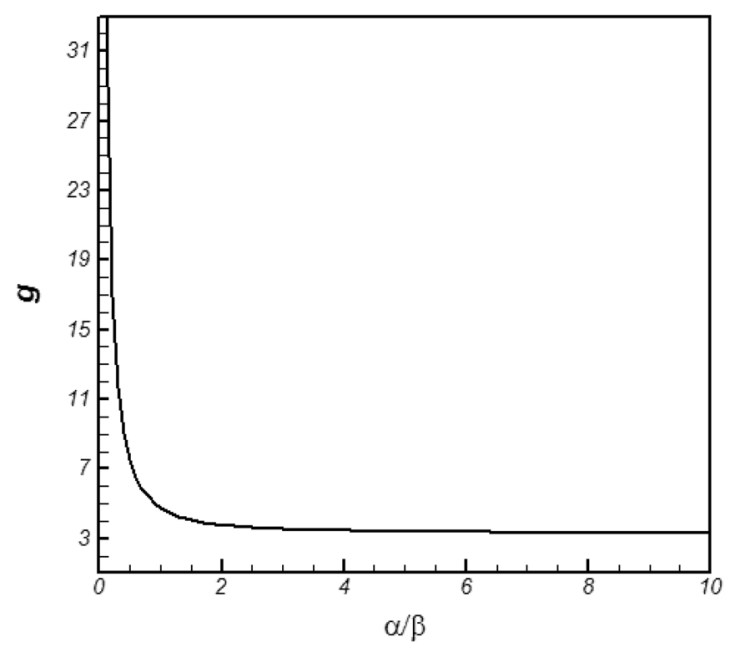

(a)

$$
Q_{\text {steady }}^{*}=V^{*} \sqrt{L_{p}^{*} \alpha \beta f}\left(\frac{\alpha}{\beta}\right) \eta\left(\frac{H a}{\left(H a_{o p t}\right)_{D C}}\right)
$$

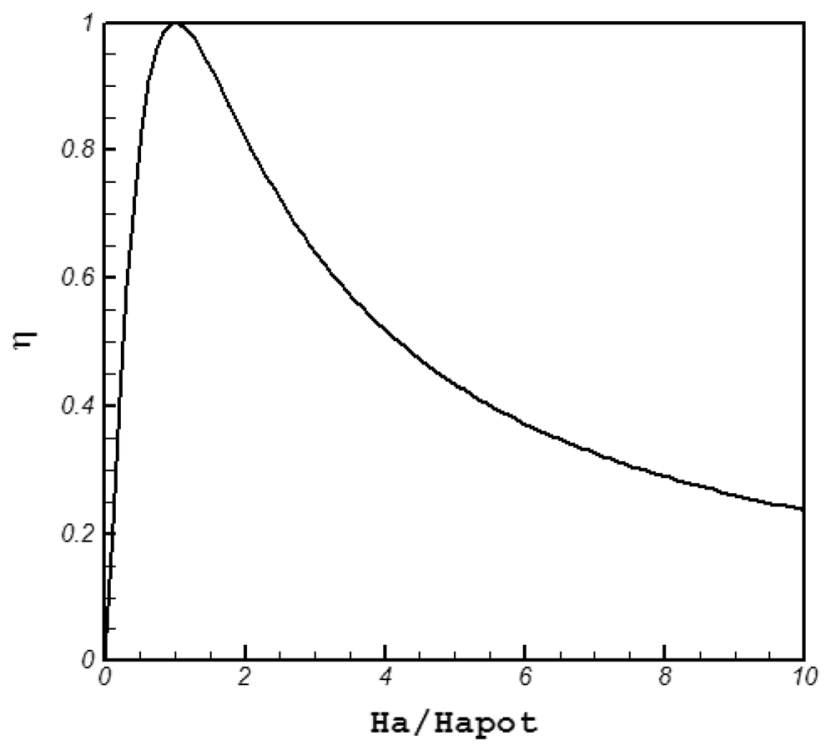

Fig. 6 variations of $\eta$ as a function of $\frac{\mathrm{Ha}}{\left(\mathrm{Ha}_{\mathrm{Opt}}\right)_{D C}}$

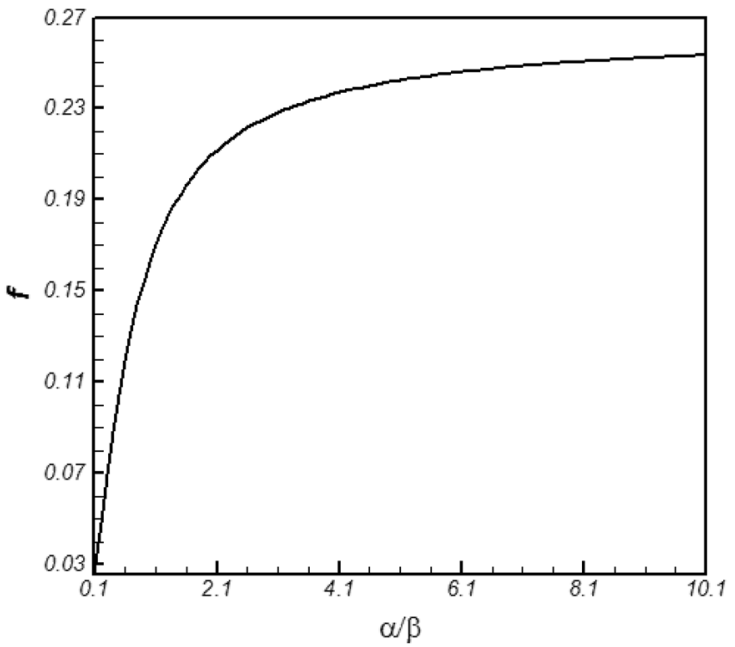

(b)

Fig. 5 variations of $f$ and $g$ as a function of $\alpha / \beta$ 


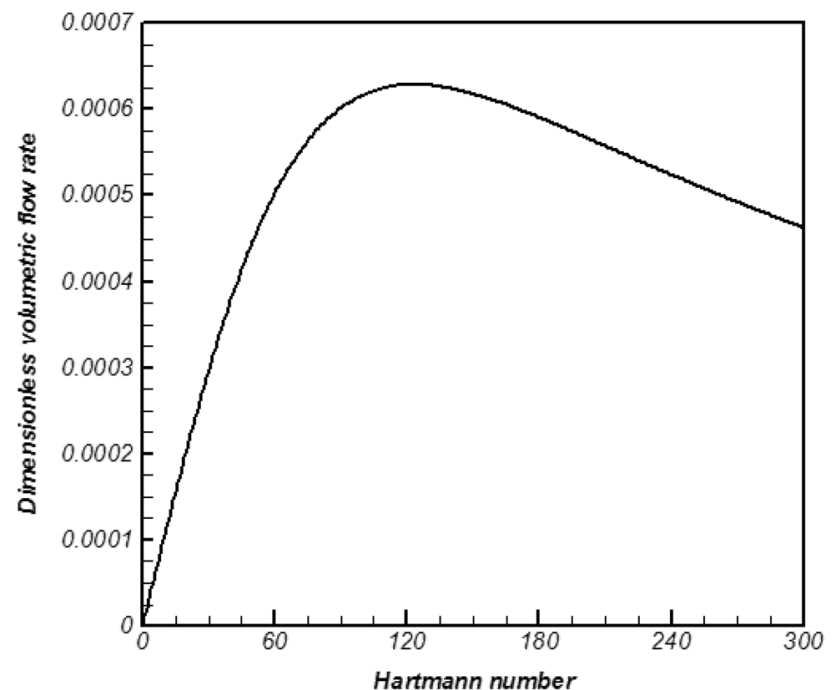

Fig. 7 Effect of the Hartmann number on the volumetric flow rate of AC-operated MHD micropump. $\left(\alpha=0.1, \quad \beta=0.1, L_{p}^{*}=0.3, V^{*}=1, \omega^{*}=10000, \phi=0\right)$

\subsubsection{Ac-operated mhd micropump}

The impact of the Hartmann number on the volumetric flow rate profile is illustrated in Fig. 7. Based on this figure, the impact of the Hartmann number in the AC-operated MHD micropump is similar to DC-operated.

Optimum Hartmann number of AC-operated MHD micropump can be found by deriving Eq. 38 :

$\frac{d Q_{\text {steady }}^{*}}{d H a}=0$

and obtain,

$\sum_{m=1}^{\infty} \sum_{n=1}^{\infty} \frac{\lambda_{m n}^{2}-\frac{L_{p}^{*} H a^{2}}{2}}{(m n)^{2}\left(\lambda_{m n}^{2}+\frac{L_{p}^{*} H a^{2}}{2}\right)^{2}}=0, \quad m$ and $n$ odd

After mathematical calculation we can derive the below equation:
$\left(H a_{o p t}\right)_{A C}=\frac{\sqrt{2}}{\beta \sqrt{L_{p}^{*}}} g\left(\frac{\alpha}{\beta}\right)$

where $g\left(\frac{\alpha}{\beta}\right)$ is plotted in Fig. 5a. Substituting Eq. 49 into Eq. 38 yields the maximum flow rate as:

$Q_{\max }^{*}=\frac{\cos \phi}{\sqrt{2}} V^{*} \sqrt{L_{p}^{*}} \alpha \beta f\left(\frac{\alpha}{\beta}\right)$

where $f\left(\frac{\alpha}{\beta}\right)$ is plotted in Fig. 5b. We can also define the efficiency of AC-operated MHD micropumps by:

$\eta\left(\frac{H a}{\left(H a_{o p t}\right)_{A C}}\right)=\frac{Q^{*}}{Q_{\max }^{*}}$

where $\eta\left(\frac{H a}{\left(H a_{o p t}\right)_{A C}}\right)$ is exactly the same as $\eta\left(\frac{H a}{\left(H a_{o p t}\right)_{D C}}\right)$ which is plotted in Fig. 6. Finally, the relation of steady volumetric flow rate is obtained as:

$Q_{\text {steady }}^{*}=\frac{\cos \phi}{\sqrt{2}} V^{*} \sqrt{L_{p}^{*}} \alpha \beta f\left(\frac{\alpha}{\beta}\right) \eta\left(\frac{H a}{\left(H a_{o p t}\right)_{A C}}\right)$

\subsubsection{Comparison of flow rates and hartmann numbers between ac- and dc-operated mhd micropump}

In Eqs. 53 and 54 the ratios of optimum Hartmann number and maximum volumetric flow rate in AC- and DCoperated MHD micropump are calculated. The maximum volumetric flow rate of DC-operated is larger than ACoperated, but the optimum Hartmann number for DCoperated MHD micropump is less than AC-operated MHD micropump. Therefore due to less optimum Hartmann number and larger maximum flow rate in DC-operated MHD micropump, it is better than AC-operated. But in the DC-operated MHD micropump, gas bubbles are produced by the electrolytic reaction and impede flow of fluid and causes dissolving of electrodes. This restricts the practi-

$\sum_{m=1}^{\infty} \sum_{n=1}^{\infty} \frac{\left(\frac{n \pi \beta}{\alpha}\right)^{2}+(m \pi)^{2}-\left(\frac{\beta \sqrt{L_{p}^{*} H a}}{\sqrt{2}}\right)^{2}}{(m n)^{2}\left(\left(\frac{n \pi \beta}{\alpha}\right)^{2}+(m \pi)^{2}+\left(\frac{\beta \sqrt{L_{p}^{*} H a}}{\sqrt{2}}\right)^{2}\right)^{2}}=0, \quad m$ and $\mathrm{n}$ odd

The Eq. 48 is similar to Eq. 41, so the relation of optimum Hartmann number for AC-operated MHD micropump is: cal application of DC-operated MHD micropump to nonelectrolytic conductors, like liquid metals. In an AC-operated MHD micropump, ac current with sufficiently high 


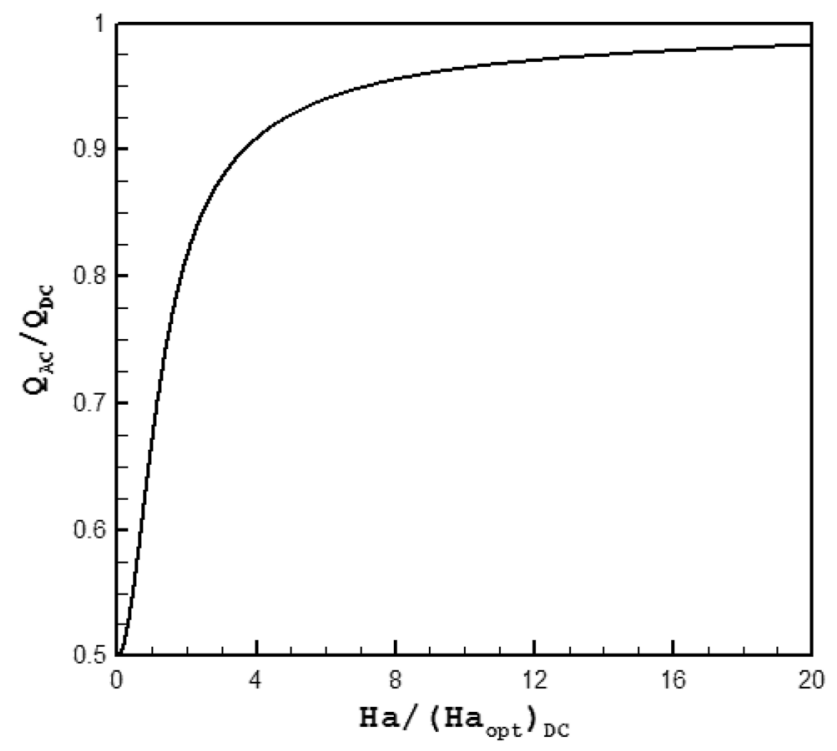

Fig. 8 variations of $\frac{\left(Q^{*}\right)_{A C}}{\left(Q^{*}\right)_{D C}}$ as a function of $\frac{H a}{\left(H a_{o p t}\right)_{D C}} \cdot(\phi=0)$

frequency causes reversal of chemical reactions occur quickly enough that no bubbles get an opportunity to form and degradation of electrode never can happen [4].

$$
\begin{aligned}
& \frac{\left(H a_{o p t}\right)_{A C}}{\left(H a_{o p t}\right)_{D C}}=\sqrt{2} \\
& \frac{\left(Q_{\max }^{*}\right)_{A C}}{\left(Q_{\max }^{*}\right)_{D C}}=\frac{\cos \phi}{\sqrt{2}}
\end{aligned}
$$

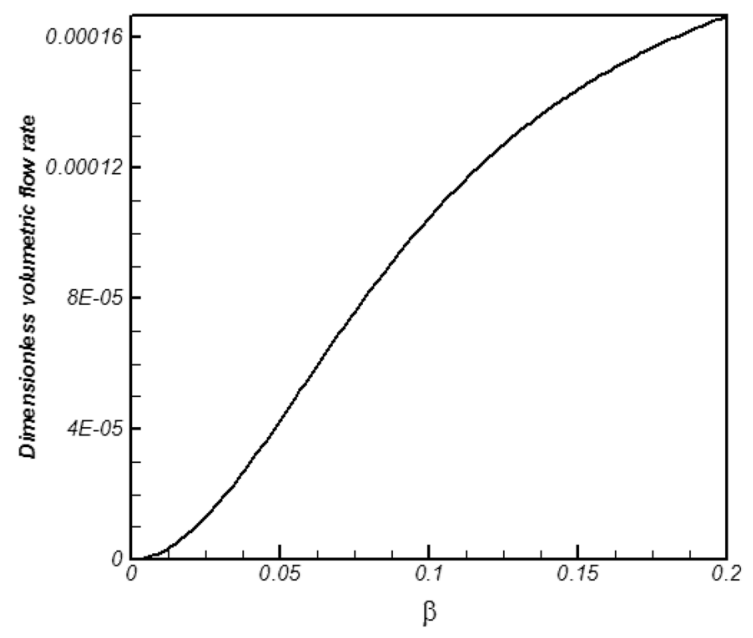

(a)
Finally the ratio of flow rates in AC- and DC-operated MHD micropump are related together in Eq. 55. Figure 8 shows the variations of $\frac{\left(Q^{*}\right)_{A C}}{\left(Q^{*}\right)_{D C}}$ as a function of $\frac{\mathrm{Ha}}{\left(\mathrm{Ha}_{\text {opt }}\right)_{D C}}$ based on this relation. This figure shows the ratio of $\frac{\left(Q^{\circ}\right)_{C C}}{\left(Q^{*}\right)_{D C}}$ is 0.5 in low values of $\frac{\mathrm{Ha}}{\left(\mathrm{Ha}_{\mathrm{opt}}\right)_{D C}}$, and approaches 1 with increasing of $\frac{\mathrm{Ha}}{\left(\mathrm{Ha} a_{\mathrm{opt}}\right)_{\mathrm{DC}}}$.

$$
\frac{\left(Q_{\text {steady }}^{*}\right)_{A C}}{\left(Q_{\text {steady }}^{*}\right)_{D C}}=\frac{\cos \phi}{\sqrt{2}} \times \frac{\eta\left(\frac{H a}{\left(H a_{o p t}\right)_{A C}}\right)}{\eta\left(\frac{H a}{\left(H a_{o p t}\right)_{D C}}\right)}
$$

\subsection{Channel's width effect}

\subsubsection{Dc-operated mhd micropump}

Figure 9 shows the impact of channel's width $(\beta)$ on the volumetric flow rate and centerline velocity profiles. It is seen that the volumetric flow rate increases with increasing channel's width but in the profile of centerline velocity, there is a maximum point. So the increasing of the volumetric flow rate after the optimum channel's width which causing maximum velocity is just for increasing volume of channel. We have also plotted the average velocity in Fig. 10. As expected, the average velocity has a maximum point.

For finding the optimum channel's width, first the relation of average velocity will be found:

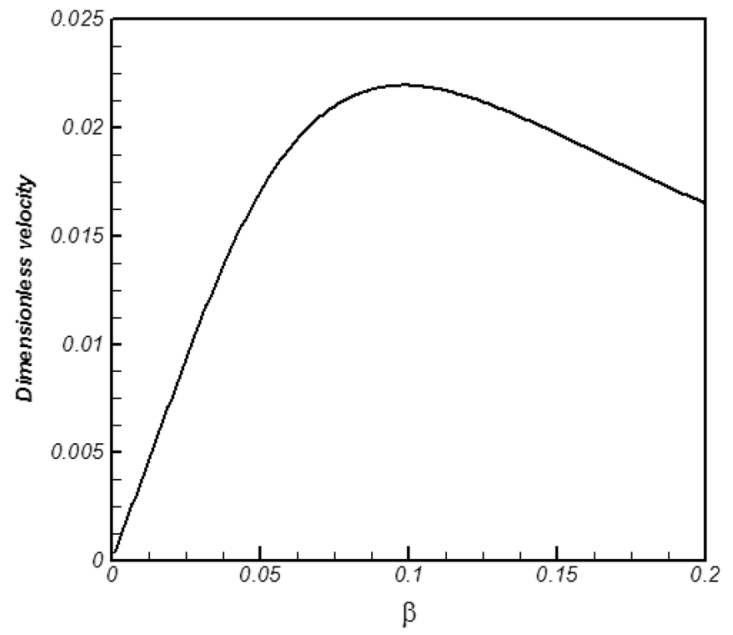

(b)

Fig. 9 Effect of the channel's width $(\beta)$ on: $\mathbf{a}$ the volumetric flow rate profile, and $\mathbf{b}$ the centerline velocity profile, in the DC-operated MHD micropump. $\left(\alpha=0.1, L_{p}^{*}=0.3, V^{*}=1, H a=5\right)$ 


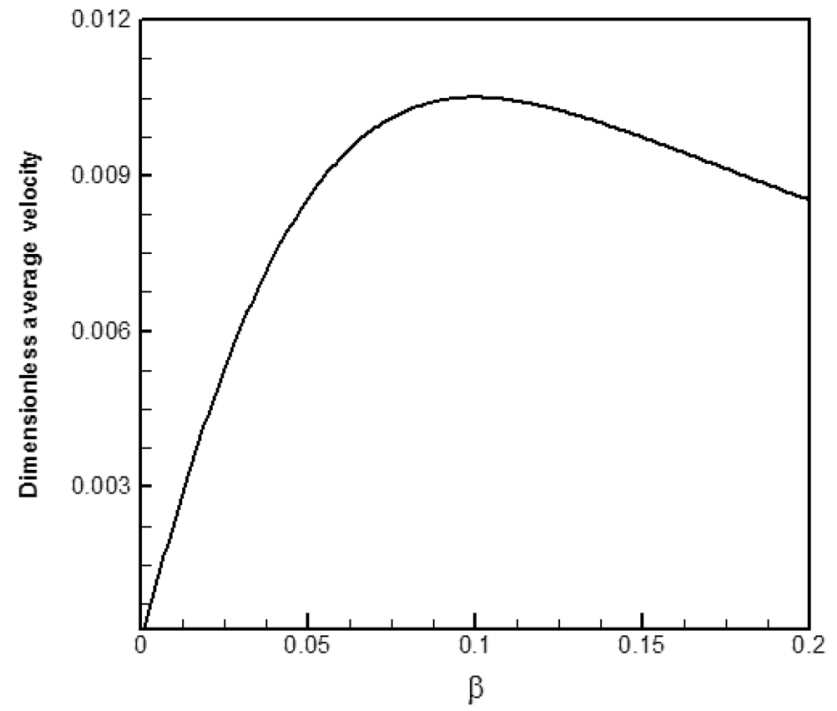

Fig. 10 Effect of the channel's width $(\beta)$ on the average velocity of DC-operated MHD micropump. $\left(\alpha=0.1, L_{p}^{*}=0.3, V^{*}=1, H a=5\right)$

${\overline{u^{*}}}_{\text {steady }}=\frac{Q_{\text {steady }}^{*}}{\alpha \times \beta}=\sum_{m=1}^{\infty} \sum_{n=1}^{\infty} \frac{128}{(m n)^{2} \pi^{4}} \times \frac{L_{p}^{*} H_{a V}^{*}}{\beta\left(\lambda_{m n}^{2}+L_{p}^{*} H^{2}\right)}, \quad \mathrm{m}$ and $\mathrm{n}$ odd

$\frac{d \overline{u^{*}} \text { steady }}{d \beta}=0$

Using numerical solution we can find $h\left(\alpha \sqrt{L_{p}^{*}} \mathrm{Ha}\right)$ which is plotted in Fig. 11. Equation 60 shows there is a relation for aspect ratio $\left(\frac{\beta}{\alpha}\right)$ which the flow rate is maximum.

From this figure, one can conclude that for $\alpha \sqrt{L_{p}^{*}} H a \prec 0.5$ (which is more relevant to electrolyte solutions) the value of $h$ is approximately 1 , so the value of aspect ratio for this state is 1 . Then we conclude that "for maximizing volumetric flow rate in electrolyte solutions, the shape of channel's cross-section should be square." From Fig. 11, another can conclude that for large values of

After mathematical calculation we can derive the below equation:

$\sum_{m=1}^{\infty} \sum_{n=1}^{\infty} \frac{\left(\frac{m \pi \alpha}{\beta}\right)^{2}-\left(\alpha \sqrt{L_{p}^{*}} H a\right)^{2}-(n \pi)^{2}}{(m n)^{2}\left(\left(\frac{m \pi \alpha}{\beta}\right)^{2}+(n \pi)^{2}+\left(\alpha \sqrt{L_{p}^{*}} H a\right)^{2}\right)^{2}}=0, \quad \mathrm{~m}$ and $\mathrm{n}$ odd

So we can find the relation of optimum channel's width as below:

$\beta_{\text {opt }}=\alpha \times h\left(\alpha \sqrt{L_{p}^{*}} H a\right)$ $\alpha \sqrt{L_{p}^{*}} H a$ (which is more relevant to liquid metals) the value of $h$ is always less than 1. Then we conclude that "for maximizing volumetric flow rate in liquid metals, the channel's width should be less than the channel's height." 


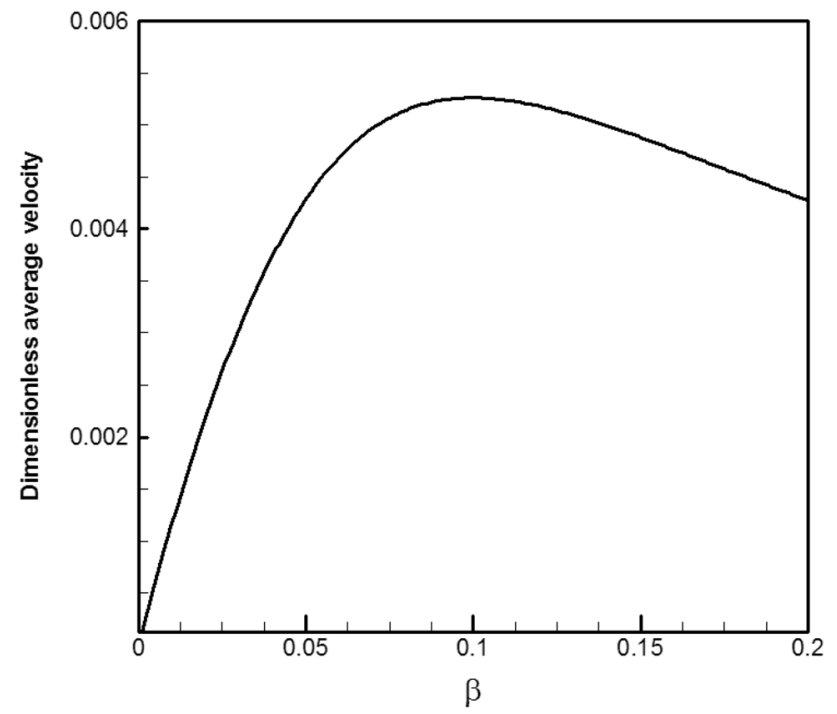

Fig. 12 Effect of the channel's width $(\beta)$ on the average velocity of AC-operated MHD micropump $\left(\alpha=0.1, L_{p}^{*}=0.3, V^{*}=1, \quad H a=5, \omega^{*}=10,000, \phi=0\right)$

\subsubsection{Ac-operated mhd micropump}

The impact of the channel's width on the average velocity is illustrated in Fig. 12. As can be seen in this figure, the effect of the channel's width in the AC-operated is similar to DC-operated.

For finding the optimum channel's width, first the relation of average velocity will be found:

${\overline{u^{*}}}_{\text {steady }}=\frac{Q_{\text {steady }}^{*}}{\alpha \times \beta}=\sum_{m=1}^{\infty} \sum_{n=1}^{\infty} \frac{64 \cos \phi}{(m n)^{2} \pi^{4}} \times \frac{L_{p}^{*} H^{*}{ }^{*}}{\beta\left(\lambda_{m n}^{2}+\frac{L_{p}^{*} H a^{2}}{2}\right)}$,

$\mathrm{m}$ and $\mathrm{n}$ odd

So:

$\sum_{m=1}^{\infty} \sum_{n=1}^{\infty} \frac{\lambda_{m n}^{2}-\frac{L_{p}^{*} H a^{2}}{2}-2\left(\frac{n \pi}{\alpha}\right)^{2}}{(m n)^{2}\left(\lambda_{m n}^{2}+\frac{L_{p}^{*} H a^{2}}{2}\right)^{2}}=0, \quad m$ and $\mathrm{n}$ odd

Then:

After mathematical calculation we can derive the below equation:

$\sum_{m=1}^{\infty} \sum_{n=1}^{\infty} \frac{\left(\frac{m \pi \alpha}{\beta}\right)^{2}-\left(\frac{\alpha \sqrt{L_{p}^{*} H}+a}{\sqrt{2}}\right)^{2}-(n \pi)^{2}}{(m n)^{2}\left(\left(\frac{m \pi \alpha}{\beta}\right)^{2}+(n \pi)^{2}+\left(\frac{\alpha \sqrt{L_{p}^{*} H a}}{\sqrt{2}}\right)^{2}\right)^{2}}=0, \quad \mathrm{~m}$ and $\mathrm{n}$ odd

So we can find the relation of optimum channel's width as below: 
$\beta_{o p t}=\alpha \times h\left(\frac{\alpha \sqrt{L_{p}^{*} H a}}{\sqrt{2}}\right)$

where the variation of $h$ versus $\frac{\alpha \sqrt{L_{p}^{*} H a}}{\sqrt{2}}$ is exactly similar to variation of $h$ versus $\alpha \sqrt{L_{p}^{*}}$ Ha which is plotted in Fig. 11 . Also we can repeat the results of DC-operated MHD micropump for AC-operated MHD micropump which: "for maximizing volumetric flow rate in electrolyte solutions, the shape of channel's cross-section should be square." and "for maximizing volumetric flow rate in liquid metals, the channel's width should be less than the channel's height."

\subsubsection{Comparison of channel's widths between ac- and dc-operated mhd micropump}

At last we compare the optimum channel's width in DCand AC-operated MHD micropump:

$\frac{\left(\beta_{\text {opt }}\right)_{A C}}{\left(\beta_{\text {opt }}\right)_{D C}}=\frac{h\left(\frac{\alpha \sqrt{L_{p}^{*} H a}}{\sqrt{2}}\right)}{h\left(\alpha \sqrt{L_{p}^{*} H a}\right)}$

Equation 66 is plotted in Fig. 13. As can be seen in this figure for values of $\alpha \sqrt{L_{p}^{*}} H a<0.5$ the values of $\beta_{\text {opt }}$ for DCand AC-operated are identical and by increasing $\alpha \sqrt{L_{p}^{*}} \mathrm{Ha}$ the value of $\beta_{\text {opt }}$ for $\mathrm{AC}$-operated become larger than DC-operated.

\section{Conclusions}

These points can be concluded on the basis of the findings presented in previous sections:

1. There is an optimum Hartmann number in both DC and AC MHD micropumps for which the volumetric flow rate is maximum.

2. The optimum Hartmann number depends on the aspect ratios of channel.

3. The maximum value of volumetric flow rate in ACoperated MHD micropump is less than the one in DCoperated.

4. The ratio of $\frac{\left(Q^{*}\right)_{A C}}{\left(Q^{*}\right)_{D C}}$ is 0.5 in low values of $\frac{H a}{\left(H a_{o p t}\right)_{D C}}$, and approaches 1 with increase of $\frac{\mathrm{Ha}}{\left(\mathrm{Ha}_{\mathrm{opt}}\right)_{D C}}$.
5. For maximizing volumetric flow rate in electrolyte solutions, the shape of channel's cross-section should be square.

6. For maximizing volumetric flow rate in liquid metals, the channel's width should be less than the channel's height.

\section{Compliance with ethical standards}

Conflict of interest The author declares that he has no conflict of interest.

\section{References}

1. Laser DJ, Santiago JG (2004) A review of micropumps. J Micromech Microeng 14:R35-R64

2. Ramos A (2007) Electrohydrodynamic and Magnetohydrodynamic. In: Hardt S, Schonfeld F (eds) Microfluidic technologies for miniaturized analysis systems, vol 2. Springer, Berlin

3. Jang J, Lee SS (2000) Theoretical and experimental study of MHD (magnetohydrodynamic) micropump. Sensors Actuators 80:84-89

4. Lemoff AV, Lee AP (2000) An AC magnetohydrodynamic micropump. Sens. Actuators B 63:178-185

5. Zhong J, Yi M, Bau HH (2002) Magneto hydrodynamic (MHD) pump fabricated with ceramic tapes. Sens. Actuators 96:59-66

6. Homsy A, Koster S, Eijkel JCT, Ven der Berg A, Lucklum F, Verpoorte E, de Rooij NF (2007) A high current density DC magneto-hydrodynamic (MHD) micro-pump. The Royal Society of Chemistry, Lab Chip, vol 5, pp 466-477

7. Eijkel J, Dalton C, Hayden C, Burt J, Manz A (2003) A circular AC magneto-hydrodynamic micro-pump for chromatographic applications. Sens. Actuators 92:215-221

8. Bau HH, Zhong J, Yi M (2001) A minute magneto hydro dynamic (MHD) mixer. Sens. Actuators B 79:205-213

9. Bau HH, Zhu J, Qian S, Xiang Y (2003) A magneto-hydrodynamically controlled fluidic network. Sens. Actuators B 88:205-216

10. Iverson Brian D, Garimella Suresh V (2008) Recent advances in microscale pumping technologies: a review and evaluation. Microfluid Nanofluid 5:145-174

11. Ho JE (2007) Characteristic study of MHD pump with channel in rectangular ducts. J Mar Sci Technol 15(4):315-321

12. Aguilar ZP, Arumugam P, Fritsch I (2006) Study of magnetohydrodynamic driven flow through LTCC channel with self-contained electrodes. J Electroanal Chem 591:201-209

13. Duwairi HM, Abdullah M (2007) Thermal and flow analysis of a magneto-hydrodynamic micro-pump. Micro-system Technol 13(1):33-39

14. Affanni A, Chiorboli G (2006) Numerical modelling and experimental study of an AC magnetohydrodynamic micropump. In: Instrumentation and measurement technology conference, Sorrento, Italy

15. Arumugam PU, Fakunle ES, Anderson EC, Evans SR, King KG, Aguilar ZP, Carter CS, Fritsch I (2006) Redox magnetohydrodynamics in a microfluidic channel: characterization and pumping. J Electrochem Soc 153:E185-E194

16. Huang L, Wang W, Murphy MC (1999) A lumped-parameter model for a micro-pump based on the magnetohydrodynamic 
(MHD) principle. In: Proceedings of SPIE-design test microfabric. MEMS MOEMS, vol 3680, pp 379-387

17. Huang L, Wang W, Murphy MC, Lian K, Ling ZG (2000) LIGA fabrication and test of a dc type magneto-hydrodynamic (MHD) micro-pump. Microsyst Technol 6:235-240

18. Moghaddam S (2013) MHD micropumping of power-law fluids: a numerical solution. Korea-Australia Rheology Journal 25(1):29-37

19. Moghaddam S (2012) Analytical solution of MHD micropump with circular channel. Int J Appl Electromagnet Mech 40:309-322

20. Kim CT, Lee J, kwon S (2014) Design, fabrication, and testing of a DC MHD micropump fabricated on photosensitive glass. Chem Eng Sci 117:210-216

21. Lim S, Choi B (2009) A study on the MHD (magnetohydrodynamic) micropump with side-walled electrodes. J Mech Sci Technol 23:739-749

22. Derakhshan S, Yazdani K (2016) 3D analysis of magnetohydrodynamic (MHD) micropump performance using numerical method. J Mech 32(1):55-62
23. Gao C, Jian Y (2015) Analytical solution of magnetohydrodynamic flow of Jeffrey fluid through a circular microchannel. J Mol Liq 211:803-811

24. Ito K, Takahashi T, Fujino T, Ishikawa M (2014) Influences of channel size and operating conditions on fluid behavior in a MHD micropump for micro total analysis system. J Int Counc Electr Eng 4(3):220-226

25. Zhao G, Jian Y, Chang L, Buren M (2015) Magnetohydrodynamic flow of generalized Maxwell fluids in a rectangular micropump under an AC electric field. J Magn Magn Mater 387:111-117

26. Wang PJ, Chang C-Y, Chang M-L (2004) Simulation of two-dimensional fully developed laminar flow for a magneto-hydrodynamic (MHD) pump. Biosens Bioelectron 20:115-121

Publisher's Note Springer Nature remains neutral with regard to jurisdictional claims in published maps and institutional affiliations. 